\title{
CONSTRUÇÃO DE ALGORITMO FUZZY PARA RASTREAMENTO SOLAR COM PAINEL FOTOVOLTAICO MONTADO EM SUPORTE DE UM EIXO
}

\author{
Willian Aparecido Leoti Zanetti ${ }^{1}$, Mario Mollo Neto $^{2}$.
}

1- $\quad$ Discente do Curso de Engenharia de Biossistemas da Faculdade de Ciências e Engenharia da Universidade Estadual Paulista Júlio de Mesquita Filho, UNESP, Campus de Tupã/SP - Brasil

2- $\quad$ Prof. Dr. da Faculdade de Ciências e Engenharia da Universidade Estadual Paulista Júlio de Mesquita Filho, UNESP, Campus de Tupã/SP - Brasil mariomollo@tupa.unesp.br

Recebido em: 03/10/2016 - Aprovado em: 21/11/2016 - Publicado em: 05/12/2016 DOI: 10.18677/EnciBio_2016B_151

\section{RESUMO}

Com o crescimento da população, a necessidade do uso de energia, vem aumentando gradativamente com o passar dos anos, conforme a dependência de energia elétrica. Esse aumento se torna mais presente, devido a muitas fontes de energia serem provenientes de combustíveis fósseis e de sistemas não sustentáveis de usinas, o que gera emissões de gases poluentes na atmosfera. Com essas questões envolvidas, aumentam as apostas para a investigação e análise do uso e importância das energias renováveis. Assim a aplicação de pesquisas para o uso de energia fotovoltaica vem crescendo, com relação à redução de custos e aplicações de sistemas fotovoltaicos com maior eficiência. De maneira geral, se tem uma necessidade em aplicação de novos materiais, otimização na fabricação das placas solares e aplicação de métodos para uma maior eficiência. Neste trabalho buscou-se o desenvolvimento da construção das funções de pertinência e a geração de uma base de regras para um sistema de inferência Fuzzy, que permita, a partir de sinais provenientes de sensores de luz, obter sinais que, futuramente, alimentem de dados a construção de um sistema de rastreamento solar automático. $O$ desenvolvimento do controlador fuzzy associado ao sistema rastreador, pode permitir uma melhor absorção de energia solar em toda a estrutura, possibilitando uma conversão com maior eficiência.

PALAVRAS- CHAVE: Lógica Fuzzy, Rastreamento, Sistema Fotovoltaico.

\section{FUZZY ALGORITHM CONSTRUCTION FOR SOLAR TRACKING WITH PHOTOVOLTAIC PANEL MOUNTED ON A SUPPORT WITH ONE AXIS}

\begin{abstract}
With population growth, the need for energy use is increasing over the years, as the dependence on electricity. This increase becomes more present, because many sources of energy are derived from fossil fuels and unsustainable systems of power plants, which generate emissions of polluting gases in the atmosphere. With these issues involved, increase the bets for research and analysis of the use and importance of renewable energy. Thus the application of research to the use of
\end{abstract}


photovoltaics is growing, with regard to reducing costs and applications of PV systems more efficiently. In general, we have a need for application of new materials, optimization in the manufacture of solar panels and application methods for greater efficiency. This work aimed to the development of construction of membership functions and the creation of a rule base for fuzzy inference system that allows, from signals from light sensors, obtain signals that future feed data to construction of an automatic sun tracking system. The development of the fuzzy controller associated with the tracker system can allow better absorption of solar energy throughout the structure, allowing greater efficiency in conversion.

KEYWORDS: Fuzzy Logic, Photovoltaic System, Tracking.

\section{INTRODUÇÃO}

Com o crescimento da população, e a alta demanda de consumo do desenvolvimento de sistemas tecnológicos, onde praticamente tudo necessita de uma fonte de energia, fica cada vez mais difícil pensar em ter que abrir mão de todas as vantagens tecnológicas que predominam atualmente, caso as fontes energéticas mais utilizadas no mundo não consigam acompanhar este desenvolvimento ou mesmo que entrem em extinção, já que a forte demanda por fontes fósseis possam levar estas a se esgotarem (MME, 2010; IEA, 2016).

A partir de uma análise temporal, pode-se compreender que no passado recente, de 1973 a 2003 o setor industrial consumiu 65\% mais energia, e apesar de ter havido aumento do uso de energia de fontes alternativas, sua participação está abaixo dos 4\%. (IEA, 2013). Atualmente a International Energy Agency (IEA) projeta, também, um crescimento de $24 \%$ no consumo final de energia até o ano 2035 e declara que em 2015 a capacidade de energia renovável instalada, cresceu e chegou a um nível recorde, com mais de 150 GW (IEA, 2016).

Já no Brasil, apontando na mesma direção, o Plano Nacional de Eficiência Energética (MME, 2010), foi concebido com a visão de aumentar a eficiência em utilização de energia elétrica até o ano 2030 através do fomento de medidas de economia de energia e busca de fontes alternativas.

Reforçando a tendência, atualmente, as pesquisas relacionadas com 0 desenvolvimento e aplicações de utilização das fontes renováveis vêm tomando grande avanço no ramo de pesquisa. Isto se deve aos meios usualmente utilizados para produção de energia elétrica não serem sustentáveis e apresentarem tendências para esgotamento no futuro, conforme destacado no trabalho de KALLEL et al. (2015), que analisam as cacterísticas de consumo sob o ponto de vista da demanda num sistema fotovoltáico.

A tecnologia relacionada com as fontes renováveis de produção de energia solar, devido à posição geográfica do Brasil, naturalmente favorecida, utilizando placas fotovoltaicas, é uma alternativa mais presente no dia a dia, pois a luz solar é uma fonte inesgotável e de baixo impacto ambiental. Assim, pensando em um melhor aproveitamento da energia solar, os estudos ligados a utilização desta fonte de energia vem crescendo muito nos últimos anos (CASTRO et al., 2008; MOEHLECKE \& ZANESCO, 2012).

A utilização deste modelo de geração de energia é dada por intermédio da aplicação de placas fotovotaicas que convertem a energia solar em elétrica, de modo que a instalação, na maioria dos casos, é fixa e determinada de acordo com a latitude do local em que é realizada a instalação, como apresentado nas pesquisas de LIOMNIS \& REINERIS (2016) e de BAHRAMIA et al. (2016). 
De modo geral, para obtenção da radiação solar, é necessário considerar diversos fatores, pois o caminho da radiação solar para chegar até a Terra é composto por interações nas regiões aonde passa. Assim esta energia viaja pelo espaço, entra na atmosfera, após, determinada quantidade de radiação solar é absorvida na atmosfera, nas nuvens e também pelas partículas presentes na atmosfera. Assim uma quantidade é refletida de volta para o espaço, e outra quantidade é absorvida pela superfície do planeta, podendo ser melhor compreendido este processo verificando-se a Figura 1.

Para que se desenvolva tal processo, visando uma melhor quantidade de absorção da radiação solar, devem-se considerar diversos fatores, tais como: período do ano, posição do sol, quantidade de propagação na atmosfera, a cobertura de nuvens, a refletividade da superfície e o tipo de superfície (ŞEN, 2008).

Radiação Solar

$(43)+(28)+(29)=100$

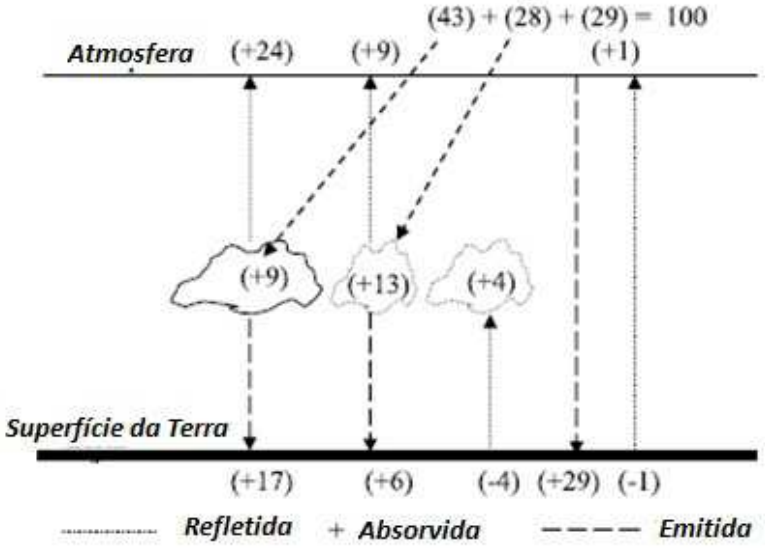

FIGURA 1. Radiação Solar Terrestre. Fonte: (ŞEN, 2008).

O processo de determinação do rendimento de um painel fotovoltaico está intimamente ligado com alguns importantes fatores. Sendo considerada a posição da placa solar em relação ao Sol, um deles. Também se apresentam outros dois fatores de influência de rendimento das placas fotovoltaicas, sendo considerados esses: o efeito da temperatura e da intensidade luminosa, pois com a intensificação da intensidade luminosa, aumenta seqüencialmente a corrente elétrica gerada pelas placas, porém a intensificação da temperatura faz com que a eficiência diminua, de modo que a operação de obtenção de máxima potência de absorção se modifique, por isso a complexidade no desenvolvimento dos projetos destes painéis e as tecnologias relacionadas aplicadas, como destacam aspectos abordados no trabalho de EBAID et al. (2013) e de VÁZQUEZ-GUZMÁN et al. (2015).

Além de se considerar o posicionamento da placa fotovoltaica em relação ao sol, também é necessário realizar a consideração do posicionamento da mesma em relação ao ângulo de inclinação (sendo este o ângulo entre o plano da superfície da placa fotovoltaica e a horizontal da sua fixação), pois está consideração pode aumentar em até $30 \%$ a absorção (FATHABADI, 2016). Os estudos realizados por WU et al. (2016), também fazem um profundo estudo matemático de modelagem de um sistema para apontamento ótimo e, estes modelos, são um bom exemplo, tanto da complexidade envolvida quanto das vantagens do processo em termos de rendimento.

Considerando a possibilidade de um melhor aproveitamento desta fonte de energia, considera-se a construção de sistemas com a utilização de rastreadores 
solares móveis, no entanto a eficiência dos mesmos é determinada pela utilização do tipo de rastreador e pela posição que o sistema de controle escolhido permite aplicar ao conjunto, o que pode ser verificado no trabalho de FATHABADI (2016).

O rastreador solar tem como funcionalidade poder captar com maior eficiência a luz solar, de modo em que seus módulos de fiação possam acompanhar a trajetória do sol desde o nascer ao repouso (Leste-Oeste). Este método de rastreamento solar deve seguir duas características fundamentais:

- Ter um seguimento de elevação solar, de modo que possa ser aplicado ajuste dos angulos de inclinação durante as mudanças sazonais;

- Aplicar um seguimento solar diário ou azimutal para a máxima incidencia de radiação solar durante o dia.

Estes fundamentos são obtidos, nos desenvolvimentos dos trabalhos de pesquisa que consideram o dimensionamento de softwares e de hardwares que fazem o rastreamento de coletores de energia solar fotovoltáicos, nos trabalhos de BARKER et al. (2013), no trabalho de YAO et al. (2014) e no trabalho de VISCONTIA et al. (2015).

Nesta pesquisa, objetivou-se a construção das funções de pertinência e a geração de uma base de regras para um sistema de inferência Fuzzy (ZADEH, 1965), que permita, a partir de sinais oriundos de sensores de luz, obter sinais que permitam, no futuro, a alimentação de dados para um sistema de controle para o rastreamento que faça automaticamente a orientação de um painel solar, buscando o máximo rendimento na conversão de energia, buscando aplicar para tal a metodologia aplicada, também, de maneira semelhante à apresentada no trabalho de pesquisa de KWAN \& WU (2016).

\section{MATERIAL E MÉTODOS}

Para o desenvolvimento deste trabalho, foi realizada inicialmente uma prospecção bibliográfica em bases nacionais e internacionais de periódicos, das quais foram obtidos os dados de fundamentação para os estudos. Foi considerada a futura construção de um protótipo com microprocessador, para suportar sensores, interfaces de potência, motores e atuadores e o painel fotovoltaico para o rastreio solar, em termos semelhantes aos propostos por BARKER et al. (2013), YAO et al. (2014) e VISCONTIA et al. (2015). Esta estrutura foi idealizada para o rastreio de um eixo, ou seja, com uma mobilidade segundo o eixo azimutal, que será capaz de orientar o módulo fotovoltaico na direção do sol ao longo do dia.

O rastreamento relativo do sol ao longo do dia será feito com auxílio da comparação do nível de tensão em dois sensores de luminosidade, do tipo Light Dependent Resistor (LDR) e aplicando-se um algoritmo de determinação das diferenças de tensão (ou erro) entre as leituras dos dois sensores. O acionamento do rastreio do painel acontecerá com a aplicação de motores controlados pelo módulo microcontrolador e as interfaces de potência.

O algoritmo de controle Fuzzy foi, então, desenvolvido utilizando-se os conceitos propostos por Lofti A. Zadeh, em 1965 na Universidade de Berkeley (ZADEH, 1965) e, os sensores irão alimentar o sistema com os indicadores das variáveis de entrada para o processo de fuzzificação, que contará com funções de pertinência triangulares e, será gerada uma base de regras para a utilização do método de inferência de Mandani para a defuzificação. A Figura 2 exibe o fluxograma do processo implementado para o experimento da presente pesquisa e teve como referência principal o terabalho de BATAYNEH et al. (2013). 
Para a construção das funções de pertinência e da base de regras, foi utilizada a ferramenta de software Matlab. $\mathrm{Na}$ sequência, realizou-se a defuzzificação, baseada em funções de pertinência de saída (também triangulares) para o acionamento dos motores para que no futuro, o sistema possa determinar o correto posicionamento para a obtenção do ponto máximo de rendimento do painel fotovoltaico.

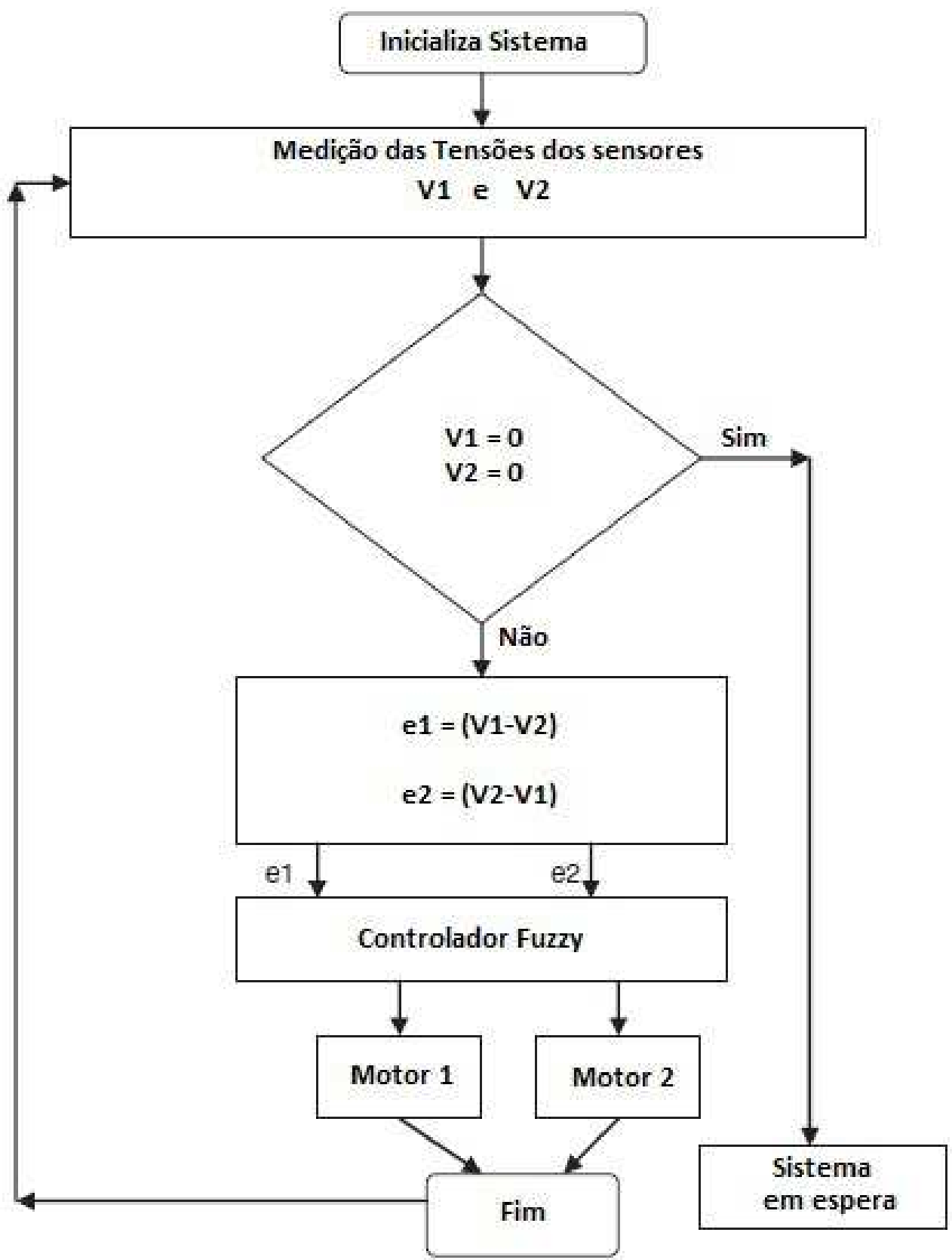

FIGURA 2. Fluxograma do sistema de detecção de erro e determinação de posicionamento do painel solar rastreador projetado. Adaptada de BATAYNEH et al. (2013). 
O controlador de lógica Fuzzy irá controlar os dois motores que moverão o painel fotovoltaico, de modo a forçá-lo a ser constantemente perpendicular à luz solar. As entradas (ou variáveis independentes) adotadas para o controlador Fuzzy foram os sinais dos erros calculados a partir das saídas dos sensores LDR. A saída do controlador Fuzzy será a variável dependente correspondente aos ângulos de giro dos dois motores.

\section{RESULTADOS E DISCUSSÕES}

O sistema foi projetado e implementado por meio de simulação digital na interface do software Matlab e as funções de pertinência obtidas para o sistema foram:

Entrada 1) Diferença obtida do número de bits lidos da porta analógica conectada ao sensor LDR 1 ( 0 a 255 bits) e do número de bits obtidos da porta analógica conectada ao sensor LDR 2 ( 0 a 255 bits). A figura 3 apresenta a função de pertinência que descreve o universo de discurso que esta variável erro 1 (e1) pode assumir e suas pertinências.

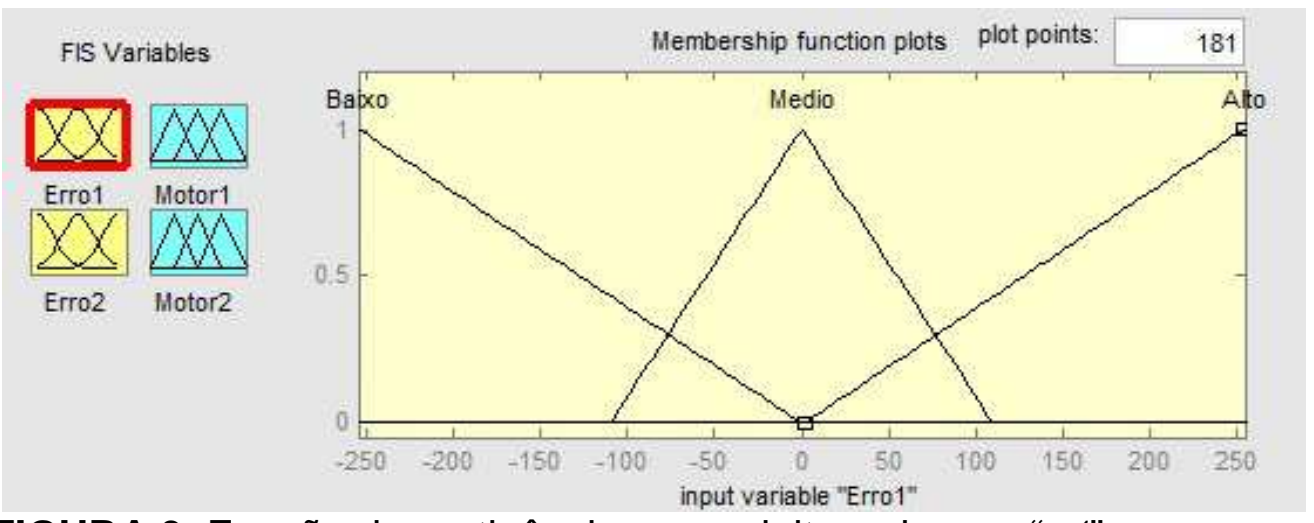

FIGURA 3. Função de pertinência para a leitura do erro "e1".

Entrada 2) Diferença obtida do número de bits lidos da porta analógica conectada ao sensor LDR 2 ( 0 a 255 bits) e do número de bits obtidos da porta analógica conectada ao sensor LDR 1 ( 0 a 255 bits). A figura 4 apresenta a função de pertinência que descreve o universo de discurso que esta variável erro 2 (e2) pode assumir e suas pertinências.

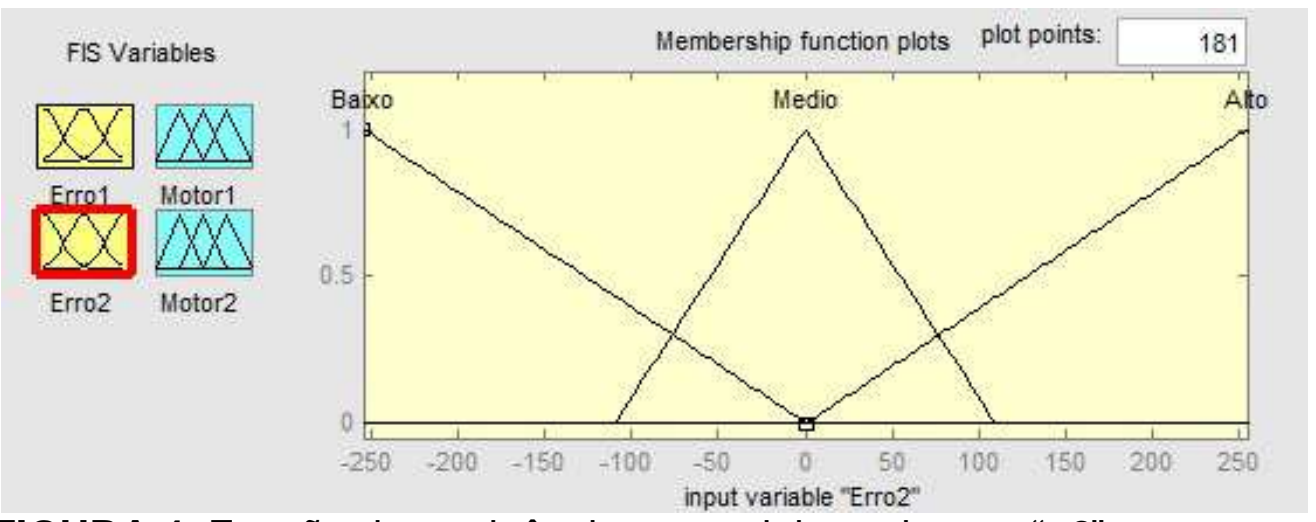

FIGURA 4. Função de pertinência para a leitura do erro "e2". 
Para as saídas do sistema, foram construídas as funções de pertinência que permitem o controle dos motores 1 e 2, considerando o seu sentido de giro (em graus) para o posicionamento do painel fotovoltaico.

Saida 1) Giro do Motor 1, controlado por motores ou servos, com suas saídas determinadas por ângulos de giro entre 0 e 180 graus. (Figura 5).

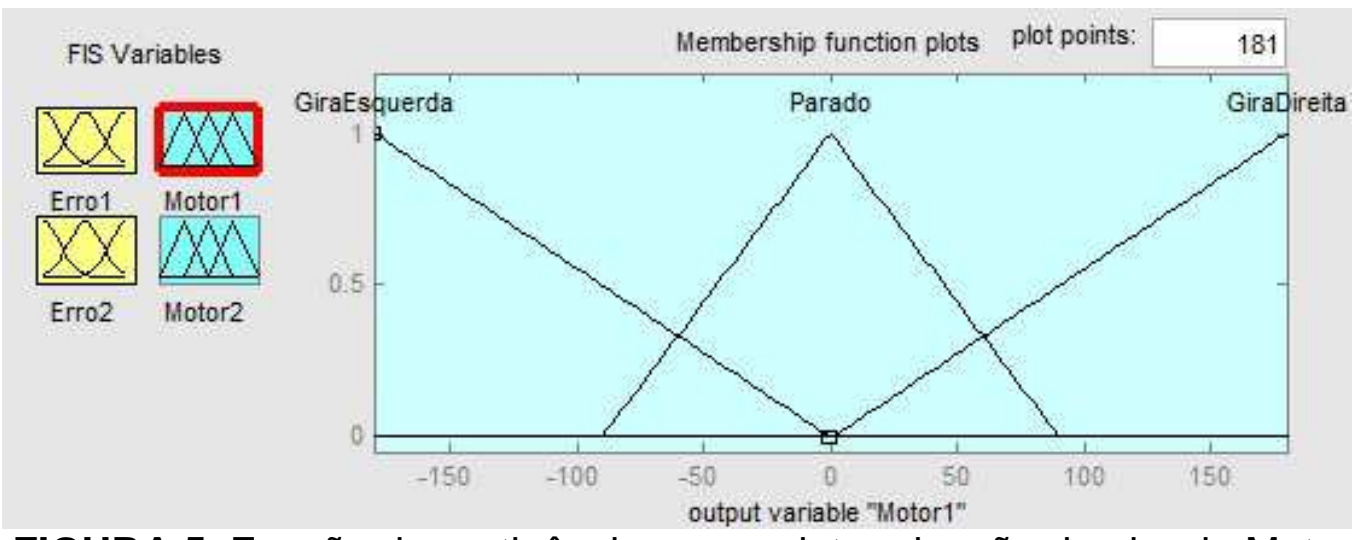

FIGURA 5. Função de pertinência para a determinação de giro do Motor 1 .

Saida 2) Giro do Motor 2, controlado por motores ou servos, com suas saídas determinadas por ângulos de giro entre 0 e 180 graus. (Figura 6).

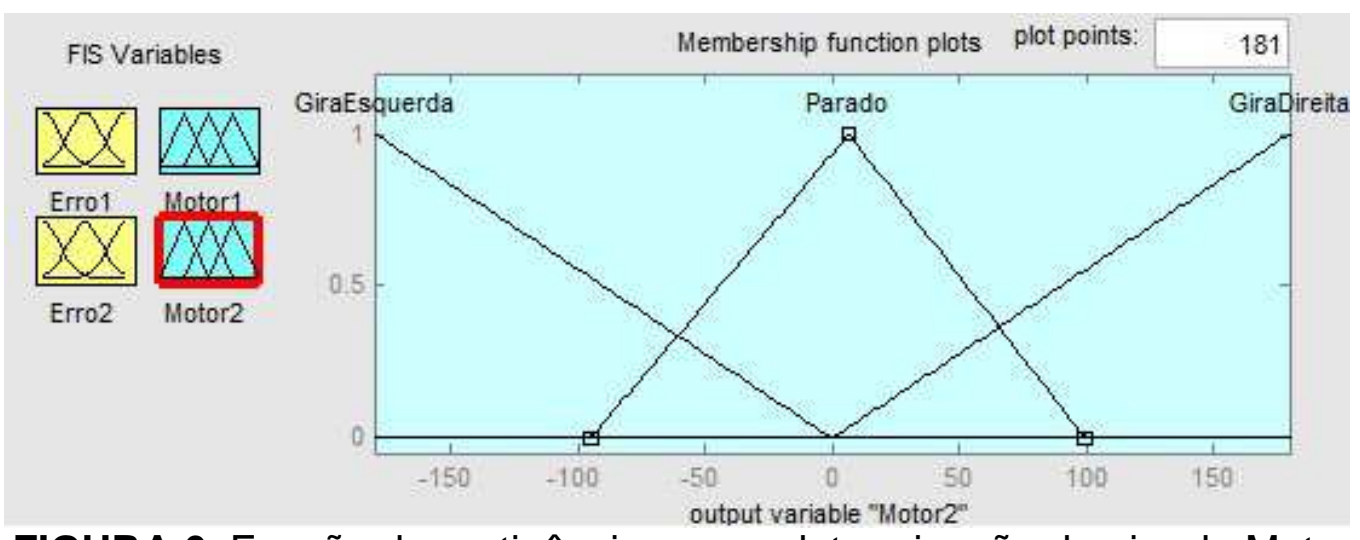

FIGURA 6. Função de pertinência para a determinação de giro do Motor 2.

A base de regras foi construída, também na interface do Matlab e ficou caracterizada com um total de 9 regras que balizam o sistema. A Figura 7 exibe a base completa, construída na interface do software Matlab.

1. If (Erro1 is Alto) and (Erro2 is Baixo) then (Motor1 is GiraDireita)(Motor2 is GiraEsquerda) (1)

2. If (Erro1 is Baixo) and (Erro2 is Alto) then (Motor1 is GiraEsquerda)(Motor2 is GiraDireita) (1)

3. If (Erro1 is Alto) and (Erro2 is Alto) then (Motor1 is GiraDireita)(Motor2 is GiraDireita) (1)

4. If (Erro1 is Baixo) and (Erro2 is Baixo) then (Motor1 is GiraEsquerda)(Motor2 is GiraEsquerda) (1)

5. If (Erro1 is Medio) and (Erro2 is Medio) then (Motor1 is Parado)(Motor2 is Parado) (1)

6. If (Erro1 is Medio) and (Erro2 is Alto) then (Motor1 is Parado)(Motor2 is GiraDireita) (1)

7. If (Erro1 is Medio) and (Erro2 is Baixo) then (Motor1 is Parado)(Motor2 is GiraEsquerda) (1)

8. If (Erro1 is Baixo) and (Erro2 is Medio) then (Motor1 is GiraEsquerda)(Motor2 is Parado) (1) 9. If (Errot is Alto) and (Erro2 is Medio) then (Mlotort is GiraDireita)(Motor2 is Parado) (1)

FIGURA 7. Base de regras que governam o sistema Fuzzy implementado. ENCICLOPÉDIA BIOSFERA, Centro Científico Conhecer - Goiânia, v.13 n.24; p.1649 
A simulação do sistema, permite visualizar os diferentes ângulos de atuação para cada um dos motores conectados à saída do sistema, de acordo com o arranjo dos cenários construídos pelas entradas provenientes dos sensores e seus respectivos valores dos erros (ver algoritmo de processamento da Figura 2).

A simulação pode ser vista na Figura 8.

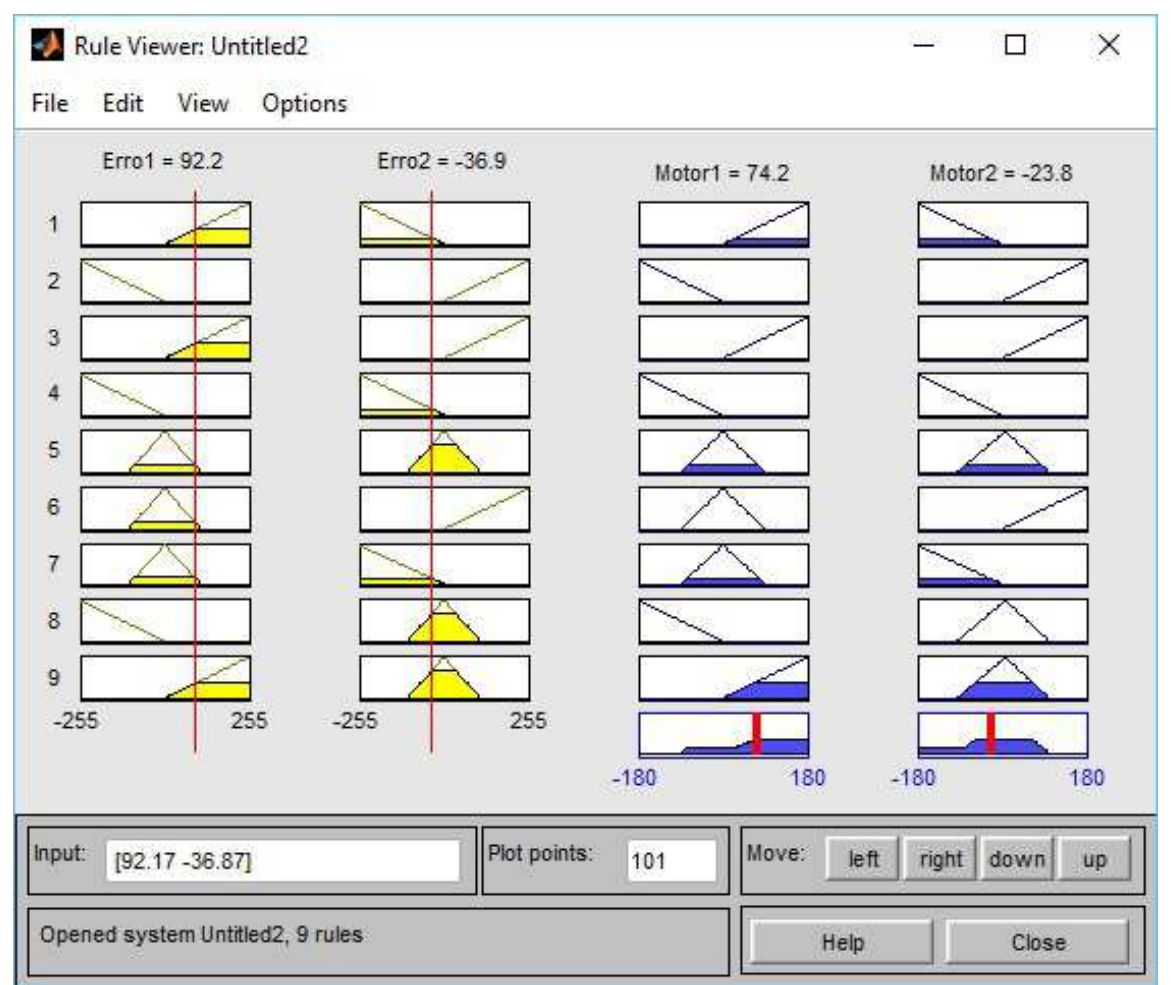

FIGURA 8. Simulação do funcionamento do sistema Fuzzy implementado.

Com esta simulação, ainda é possível a visualização das superfícies de excursão para as saídas do Motor 1 (Figura 9 a) e do Motor 2 (Figura 9 b), considerando todos os cenários de combinações possíveis para as entradas dos sensores, por meio dos valores calculados para o erro1 e erro2.
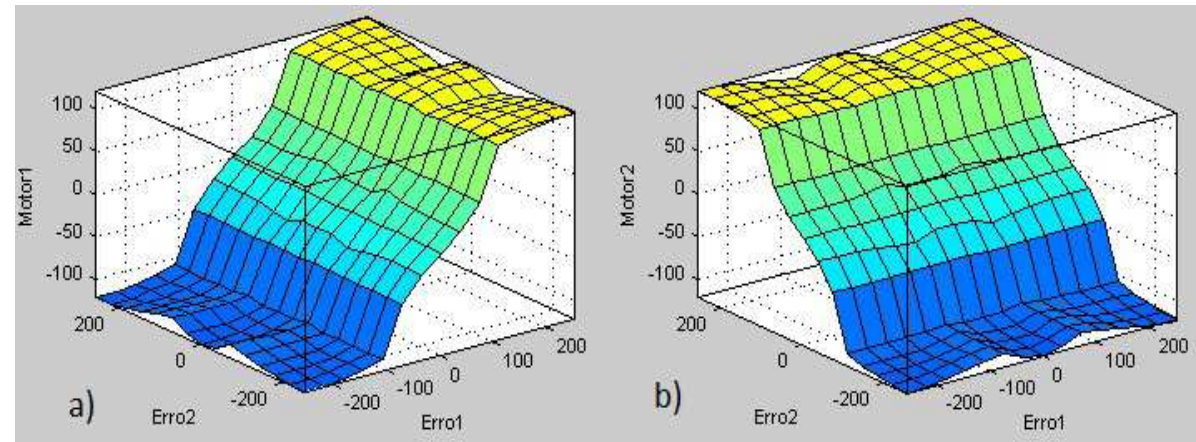

FIGURA 9. Superfícies de excursão das saídas do sistema Fuzzy implementado. a) Motor 1 e b) Motor 2.

Os resultados apresentados, baseados em simulação digital, em ambiente Matlab, visando uma melhor quantidade de absorção da radiação solar, mostram que, na construção de um sistema de rastreio com a utilização de placas ENCICLOPÉDIA BIOSFERA, Centro Científico Conhecer - Goiânia, v.13 n.24; p.1650 2016 
fotovoltáicas, pode-se aumentar em até 30\% a absorção de energia como foi indicado pelos pesquisadores FATHABADI (2016) e WU et al. (2016) em suas pesquisas.

A montagem do futuro sistema de rastreio, proposto nesta pesquisa, irá, com base neste algoritmo Fuzzy proposto, aliar a integração dos sensores e atuadores indicados nesta simulação, de forma a aproveitar mais a energia solar disponível, ao invés de efetuar-se um apontamento simples como indicam em suas pesquisas os autores LIOMNIS \& REINERIS (2016) e de BAHRAMIA et al. (2016), que consideraram simplesmente um painel solar com uma estrutura fixa e com posicionamento determinado de acordo com a latitude do local em que é realizada a instalação. Estes aprimoramentos propostos para o sistema, permitirão alcançar maior eficiência na conversão que ocorrerá nas placas fotovoltáicas. Porém, devemse, ainda, considerar diversos fatores que podem fugir ao controle do sistema, como indica o pesquisador ŞEN (2008), tais como: quantidade de propagação na atmosfera, a cobertura de nuvens, a refletividade da superfície e o tipo de superfície em que se pretende instalar o sistema.

Desta forma, o sistema apresentado que contará com estrutura idealizada para o rastreio de um eixo, ou seja, com uma mobilidade segundo o eixo azimutal, irá se comportar mais eficiente com base no sistema de controle Fuzzy, moldado conforme as indicações de ZADEH (1965), montado à partir dos sensores e atuadores, corroborando com os resultados que foram obtidos nos trabalhos de BARKER et al. (2013), no trabalho de YAO et al. (2014) e no trabalho de VISCONTIA et al. (2015). Nota-se que essa tecnologia apresentada é uma candidata a forma mais conveniente de aplicação, pois pode permitir uma maior sustentabilidade e eficiência na utilização dos painéis fotovoltáicos.

$\mathrm{O}$ algoritmo gerado pelas funções de pertinência projetadas e pela base de regras de controle geradas, deverá ser embarcado futuramente em um hardware baseado em microcontrolador, suportando os sensores, interfaces de potência, motores e atuadores, assim como o painel fotovoltáico, atendendo às prescrições encontradas nos trabalhos de BARKER et al. (2013), YAO et al. (2014) e VISCONTIA et al. (2015) garantindo uma maior eficiência energética na conversão de luz solar em energia elétrica, permitindo maior quantidade de absorção de radiação solar durante todo o período de luz solar disponível no transcorrer do dia.

Também, quando do desenvolvimento do proótipo com o sistema de controle proposto, deverão ser realizadas medições para observar o compoprtamento dos dois fatores de influência de rendimento das placas fotovoltáicas, o efeito da temperatura e da intensidade luminosa, conforme abordado nos trabalho de EBAID et al. (2013) e VÁZQUEZ-GUZMÁN et al. (2015).

Apesar do fato do retorno do investimento ainda ser considerado demorado, se comparado com os benefícios gerados a sociedade, é possível compreender que o sistema de energia solar vem se destacando entre os demais modelos de energias renováveis, que no futuro deverá ter seus componentes mais barateados, reduzindo o tempo de retorno do investimento realizado para a instalação, como pode ser visto em IEA (2016).

\section{CONCLUSÕES}

Com base nos resultados obtidos das simulações, pode-se concluir que a metodologia adotada permitiu a construção das funções de pertinência das variáveis independentes, entradas do sistema de controle, com os sinais provenientes dos 
sensores e, isto permitiu a construção das funções de pertinência para duas variáveis dependentes, correspondentes ao giro dos motores de posicionamento do painel solar, que são as saídas do sistema. A base de regras para o sistema de controle de rastreamento permite que, automaticamente, seja feita a orientação de um painel solar, buscando o máximo rendimento na conversão de energia.

Estes dados obtidos permitirão, no futuro, a construção de um protótipo microcontrolado, com o algoritmo Fuzzy desenvolvido nesta pesquisa embarcado. Com este protótipo será possível avaliar a performance do sistema de controle e observar o rendimento e eficiência do dispositivo e, o mesmo servirá de base para a construção de um sistema em dimensões reais para a utilização residencial.

\section{AGRADECIMENTOS}

Os autores agradecem ao CNPq e à UNESP pela bolsa ISB concedida para suporte à pesquisa e a unidade da UNESP, câmpus de Tupã/SP pelo suporte e disponibilização laboratórios para o desenvolvimento e de área para a realização das pesquisas bibliográficas e em laboratórios de informática para as simulações que foram necessárias ao experimento.

\section{REFERÊNCIAS}

BAHRAMIA, A.; OKOYEB, C. O.; ATIKOLA, U. The effect of latitude on the performance of different solar trackers in Europe and Africa. Applied Energy, n.1, v.177, p.896-906, 2016. Disponível em:< http://www.sciencedirect.com/science/article/pii/S0306261916307085> Acesso em: 08/09/2016. doi: http://dx.doi.org/10.1016/j.apenergy.2016.05.103

BARKER, L.; NEBER, M.; LEE, H. Design of a low-profile two-axis solar tracker. Solar Energy, n.1, v.97, P.569-576, 2013. Disponível em:< http://www.sciencedirect.com/science/article/pii/S0038092X13003757> Acesso em: 08/09/2016. doi: http://dx.doi.org/10.1016/j.solener.2013.09.014

BATAYNEH, W.; OWAIS, A.; NAIROUKH, M. An intelligent fuzzy based tracking controller for a dual-axis solar PV system. Automation in Construction, v.29, p.100-106, 2013. Disponível em:< http://www.sciencedirect.com/science/article/pii/S0926580512001550> Acesso em: 08/09/2016. doi: http://dx.doi.org/10.1016/j.autcon.2012.09.006

CASTRO, M.; ANTÓN, I.; SALA, G. Pilot production of concentrator silicon solarcells: Approaching industrialization. Solar Energy Materials \& Solar Cells, v.1, n.92 p. 1697-1705, 2008. Disponível em: < http://www.sciencedirect.com/science/article/pii/S0927024808002559> Acesso em 08/09/2016. doi: http://dx.doi.org/10.1016/j.solmat.2008.08.001

EBAID, M. S. Y.; QANDIL, H.; HAMMADB, M. A unified approach for designing a photovoltaic solar system for the underground water pumping well-34 at Disi aquifer. Energy Conversion and Management v.1, n.75, p.780-795, 2013. Disponível em: $<\quad$ http://ac.els-cdn.com/S0196890413004585/1-s2.0-S0196890413004585main.pdf?_tid=bb5b18ea-7a0b-11e6-b356-

00000aacb361\&acdnat=1473810328_81852b15cf66456e4b934bbbe03e0ad7 Acesso em: 08/09/2016. doi: http://dx.doi.org/10.1016/j.enconman.2013.07.083 
FATHABADI, H. Novel high efficient offline sensorless dual-axis solar tracker for using in photovoltaic systems and solar concentrators. Renewable Energy, n.1, v.95, p.485-494, 2016. Disponível em:< http://www.sciencedirect.com/science/article/pii/S0960148116303652> Acesso em 08/09/2016. doi: http://dx.doi.org/10.1016/j.renene.2016.04.063

IEA. International Energy Agency. Energy Technology Perspectives: Towards Sustainable Urban Energy Systems, 2016. Disponível em:< http://www.iea.org/media/etp/etp2016/160601_ETP2016_CEM7_rev.pdf> Acesso em: 13/09/2016. doi: Não Disponível.

IEA. Key World Energy Statistics. International Energy Agency, 2013.Disponível em <http://www.iea.org/publications/freepublications/publication/KeyWorld2013.pdf>, acesso em 25/05/2016. doi: Não Disponível.

KALLEL, R. ; BOUKETTAYA, G; KRICHEN, L. Demand side management of household appliances in stand-alone hybrid photovoltaic system. Renewable Energy, n.1, v. 81, p.123-135, 2015. Disponível em:< http://www.sciencedirect.com/science/article/pii/S0960148115002062> Acesso em: 08/09/2016. doi: http://dx.doi.org/10.1016/j.renene.2015.03.024

KWAN, T. H.; WU, X. Maximum power point tracking using a variable antecedent fuzzy logic controller. Solar Energy, n.1, v.137, p.189-200, 2016. Disponível em:< http://www.sciencedirect.com/science/article/pii/S0038092X16303358> Acesso em: 08/09/2016. doi: http://dx.doi.org/10.1016/j.solener.2016.08.008

LIOMNIS, O. L.;REINERIS, M. L. Análisis energético de un sistema fotovoltaico integrado a una cubierta plana horizontal. Ingeniería Energética, v.XXXVII(1):45-54, 2016. ISSN 1815-5901, Disponível em:<http://rie.cujae.edu.cu/index.php/RIE/article/view/465/495> Acesso em: 01/09/2016. doi: Não Disponível.

MME. Plano Nacional de Eficiência Energética - PNEf: 2010-2030. Ministério de Minas e Energia- Brasília - Brasil - 2010. Disponível em< http://www.orcamentofederal.gov.br/projeto-esplanada-sustentavel/pasta-paraarquivar-dados-do-pes/Plano_Nacional_de_Eficiencia_Energetica.pdf>, acesso em 27/05/2016. doi: Não Disponível.

MOEHLECKE, A.; ZANESCO, I. Development of silicon solar cells and photovoltaic modules in Brazil: analysis of a pilot production. Materials Research, São Carlos , v. $15, \quad$ n. $4, \quad$ p. $581-588,2012.2$ Disponível em: <http://www.scielo.br/scielo.php?script=sci_arttext\&pid=S151614392012000400016\&lng=en\&nrm=iso >. Acesso em 13/09/2016. doi: http://dx.doi.org/10.1590/S1516-14392012005000084

ŞEN, Z. Solar Energy Fundamental and Modeling Tecnhiques: Atmosphere, environment, climate change and renewable energy.1ed. London: Springer, 2008. 276p. ISBN 978-1-84800-134-3. doi: Não Disponível. 
VÁZQUEZ-GUZMÁN, GERARDO, PÁNFILO RAYMUNDO MARTÍNEZRODRÍGUEZ, JOSÉ MIGUEL SOSA-ZÚÑIGA. High Efficiency Single-Phase Transformer- less Inverter for Photovoltaic Applications. Ingeniería Investigación y Tecnología, v. XVI,n. 02, p. 173-184, 2015. Disponível em: < http://www.ingenieria.unam.mx/ revistafi/ejemplaresHTML/V16N2/V16N2_art02/V16 N2_art02.pdf> Acesso em 08/09/2016. http://dx.doi.org/10.1016/j.riit.2015.03.002.

VISCONTIA, P.; COSTANTINIA, P.; ORLANDOA, C.; LAY-EKUAKILLEA, A.; CAVALERAB, G. Software solution implemented on hardware system to manage and drive multiple bi-axial solar trackers by PC in photovoltaic solar plants. Measurement, n.1, v.76, p.80-92, 2015. Disponível em:< http://www.sciencedirect.com/science/article/pii/S0263224115004376> Acesso em: 08/09/2016. doi: http://dx.doi.org/10.1016/j.measurement.2015.08.024

WU, J.; ZHANG, B.; WANG, L. Optimum design and performance comparison of a redundantly actuated solar tracker and its nonredundant counterpart. Solar Energy, n.1, v.127, p.36-47, 2016. Disponível em:< http://www.sciencedirect.com/science/article/pii/S0038092X1600030X> Acesso em 08/09/2016. doi: http://dx.doi.org/10.1016/j.solener.2016.01.017

YAO, Y.; HU, Y.; GAO, S.;YANG, G.; DU, J. A multipurpose dual-axis solar tracker with two tracking strategies. Renewable Energy, n.1, v.72, p.88-98, 2014. Disponível http://www.sciencedirect.com/science/article/pii/S0960148114003887> Acesso em: 08/09/2016. doi: http://dx.doi.org/10.1016/j.renene.2014.07.002

ZADEH, L. A. Fuzzy sets - Department of Electrical Engineering and Electronics Research Laboratory, University of California. Information and Control, California. V. 8, p. 338-353, Jun 1965. Disponível em: < https://people.eecs.berkeley.edu/ zadeh/papers/Fuzzy\%20SetsInformation\%20and\%20Control-1965.pdf> Acesso em 24/08/2016. doi:10.1016/S0019-9958(65)90241-X 